\title{
Commentary: Long-term in vivo microscopy of CAR T cell dynamics during eradication of CNS lymphoma in mice
}

\author{
Hocine Rachid Hocine ${ }^{1}$, Hue T. Quach ${ }^{1}$ and Prasad S. Adusumilli ${ }^{1,2 *}$ \\ ${ }^{1}$ Thoracic Service, Department of Surgery, Memorial Sloan Kettering Cancer Center, New York, NY, United States, ${ }^{2}$ Center \\ for Cell Engineering, Memorial Sloan Kettering Cancer Center, New York, NY, United States
}

Keywords: solid tumors, blood-brain barrier, CAR T cells, regional administration, CNS lymphoma

\section{A Commentary on}

Long-term in vivo microscopy of CAR T cell dynamics during eradication of CNS lymphoma in mice

OPEN ACCESS

Edited by: John - Maher,

King's College London,

United Kingdom

Reviewed by:

Nabil Ahmed,

Baylor College of Medicine,

United States

Sonia Guedan,

August Pi i Sunyer Biomedical

Research Institute (IDIBAPS), Spain

*Correspondence:

Prasad S. Adusumilli

adusumip@mskcc.org

Specialty section:

This article was submitted to Cancer Immunity and Immunotherapy,

a section of the journal

Frontiers in Immunology

Received: 13 April 2020

Accepted: 09 June 2020

Published: 21 July 2020

Citation:

Hocine HR, Quach HT and Adusumilli PS (2020) Commentary: Long-term in vivo microscopy of CAR

$T$ cell dynamics during eradication of

CNS lymphoma in mice.

Front. Immunol. 11:1503.

doi: 10.3389/fimmu.2020.01503 by Mulazzani, M., Fräßle, S. P., von Mücke-Heim, I., Langer, S., Zhou, X., Ishikawa-Ankerhold, H., et al. (2019). Proc. Natl. Acad. Sci. U.S.A. 116:24275-24284. doi: 10.1073/pnas.1903854116

\section{INTRODUCTION}

Diffuse large B-cell lymphoma (DLBCL), an aggressive extranodal non-Hodgkin lymphoma, accounts for $90 \%$ of cases of primary central nervous system lymphoma (PCNSL). Despite treatment with chemotherapy and immunotherapy, 1 in 3 patients with DLBCL either have refractory disease or experience relapse $(1,2)$. Anti-CD19 CAR T cells have elicited dramatic responses in hematological malignancies, but their efficacy against solid tumors and hematological malignancies with intracerebral tumors remains limited. The hurdles to achieving antitumor efficacy against solid tumors using CAR T cells-such as limited infiltration, limited penetration, and an immunosuppressive microenvironment-are also impediments to eradicating intracerebral tumors in PCNSL (3). In addition, the blood-brain barrier impedes intravenous CAR Tcell administration.

\section{NOVELTY}

The authors developed a mouse model of orthotopic, fluorescent-labeled PCNSL with intracerebral and periventricular tumor growth and created chronic cranial windows, which allowed longitudinal observation of tumor and T-cell kinetics (4). Three-dimensional quantification of intratumoral T cells by use of two-photon laser scanning microscopy (TPLSM) demonstrated that intravenously administered anti-CD19 CAR T cells accumulated in limited numbers at a late time point, at levels equal to mock CAR T cells, resulting in tumor regression in a fraction of treated mice. In contrast, intracerebrally administered anti-CD19 CAR T cells accumulated and penetrated deeply at higher numbers than mock CAR T cells.

The most remarkable achievement of this study was its use of cranial windows and TPLS to observe intratumoral proliferation, CAR T-cell infiltration, and intracerebral CAR T-cell velocities at the tumor site without the need to sacrifice mice (Figure 1). It is likewise impressive that the study demonstrated that, although the non-activated CAR T-cell velocity in intracerebral 


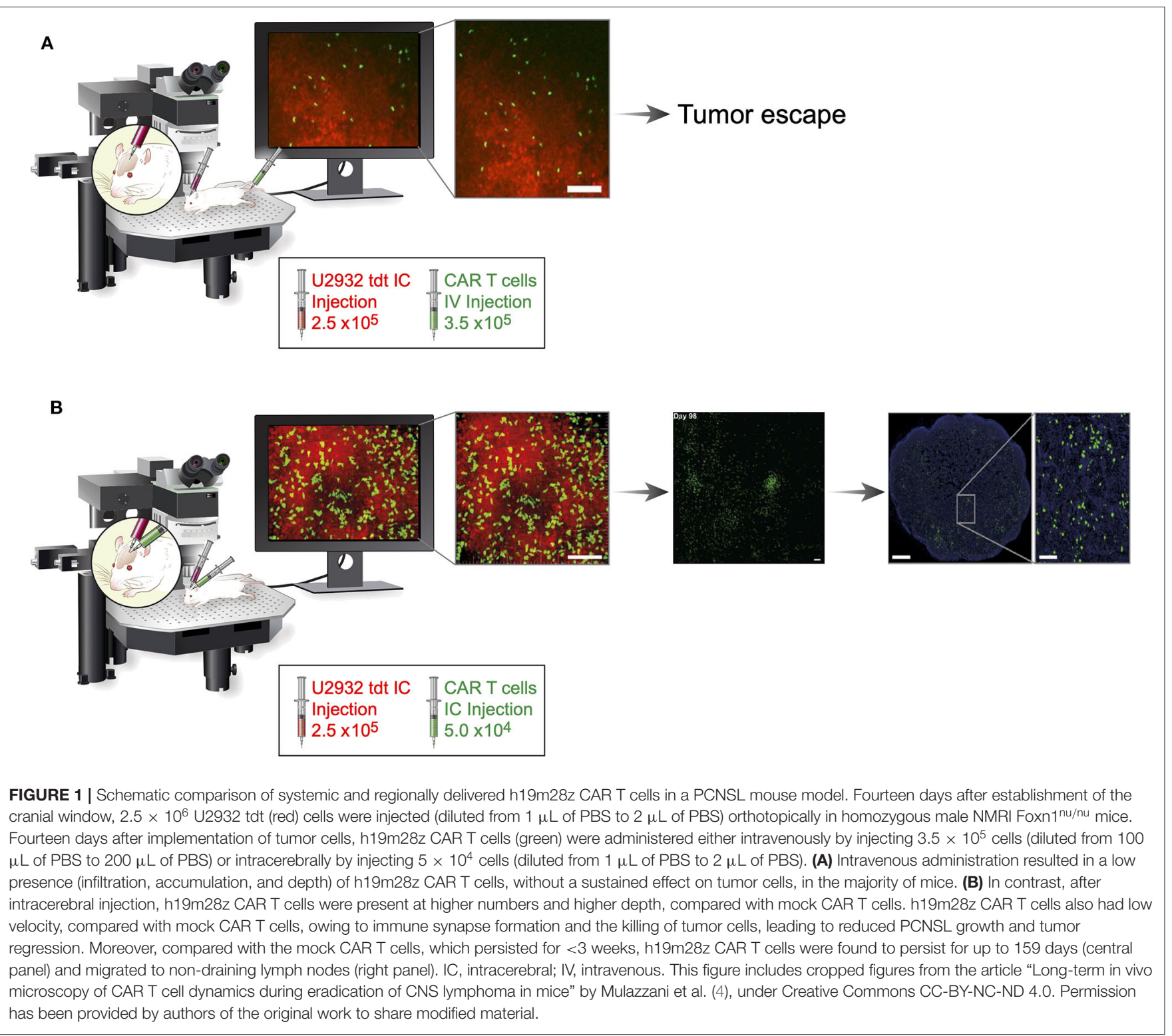

tumors remained unchanged, antigen-activated anti-CD19 CAR $\mathrm{T}$ cells had reduced velocity, compared with mock CAR $\mathrm{T}$ cells. Reduced velocities suggest CAR $\mathrm{T}$ cells form an immunological synapse with tumor cells and that, after tumor eradication, intratumoral tumor-specific CAR $\mathrm{T}$ cells may resume higher velocities-observations that were associated with tumor regression. These observations were confirmed in mice without cranial windows, indicating that surgery-related inflammation did not influence results. The authors showed a difference in infiltration patterns. Whereas mock CAR T cells primarily accumulated at the tumor borders, h19m $28 \mathrm{z}$ CAR T cells significantly outnumbered mock CAR T cells and infiltrated deeper tumor regions. Immunofluorescence analyses of harvested organs showed that anti-CD19 CAR T cells lacked $\mathrm{CD} 27$, a marker of differentiation from resting memory $\mathrm{T}$ cells to terminal effector T cells. In contrast, mock CAR T cells retained CD27. Even after tumor regression, intracerebral anti-CD19 CAR $\mathrm{T}$ cells were observed for 35 to 159 days. More importantly, following intracerebral injection, circulating anti-CD19 CAR T cells were observed in non-draining, distant inguinal lymph nodes at higher numbers than mock CAR T cells.

\section{DISCUSSION}

The study provides a comprehensive in vivo analysis of the mechanisms of CAR T cells in a PCNSL model, but the results raise questions that require further study. First, the authors showed that, in mice treated with mock CAR $\mathrm{T}$ cells, but not in mice treated with h19m28z CAR T cells, CD3 T cells were in close association with CD11c myeloid cells, which 
formed a dense rim around the tumor. The effects of tumoral stroma and the tumor immunosuppressive microenvironment on CAR T-cell migration, proliferation, and egress are not clear. These important factors that limit antitumor efficacy should therefore be studied in a solid-tumor model. The information obtained may not only highlight the influence of organ-specific immune microenvironments on CAR $\mathrm{T}$ cells but also allow investigators to identify strategies to manipulate the tumor immune microenvironment to further improve the antitumor efficacy of CAR T cells in solid tumors. Second, the authors show that the dose of CD19 CAR T cells delivered intracerebrally $\left(2 \times 10^{4}\right.$ CAR $\mathrm{T}$ cells $)$ was more effective than a 17.5fold higher dose delivered intravenously $\left(3.5 \times 10^{5}\right.$ CAR T cells). We and others have shown that, following regional administration of CAR T cells, CD4 CAR T cells are antigenactivated and can provide better helper function to CD8 CAR $\mathrm{T}$ cells (5); this results in higher antitumor efficacy, compared with systemic administration. Further dissection of the individual and interdependent roles of CD4 and CD8, as well as those of CAR and non-CAR T cells, within the solid-tumor environment following regional administration is therefore warranted. Third, it is not clear whether the entry of anti-CD19 CAR T cells into non-draining lymph nodes was influenced by cancer cells or whether the altered chemokine receptor profile of antigenactivated anti-CD19 CAR T cells facilitated their entry. A better understanding of CAR T-cell entry into tumors and lymph nodes will greatly influence solid-tumor CAR T-cell therapy. Fourth, understanding CAR T-cell dynamics in heterogenous antigen expression (6), which is common in solid tumors, unlike CD19 expression in hematological malignancies, is important. Fifth, we and others have shown that exhausted CAR T cells can be rescued by anti-PD1 strategies (6); as such, visualization of CAR T-cell kinetics following administration of anti-PD1 agent is relevant to current clinical applications (7).

The translational relevance of this study also raises questions for further investigation. Neurological toxicities, collectively referred to as CAR T cell-related encephalopathy syndrome, occur in approximately $12 \%$ to $32 \%$ of patients treated with CD19 CARs, highlighting the life-threatening risks of immune

\section{REFERENCES}

1. Bachanova V, Perales MA, Abramson JS. Modern management of relapsed and refractory aggressive B-cell lymphoma: a perspective on the current treatment landscape and patient selection for CAR Tcell therapy. Blood Rev. (2019) 40:100640. doi: 10.1016/j.blre.2019.1 00640

2. Sehn LH, Gascoyne RD. Diffuse large B-cell lymphoma: optimizing outcome in the context of clinical and biologic heterogeneity. Blood. (2015) 125:22-32. doi: 10.1182/blood-2014-05-5 77189

3. Tahmasebi S, Elahi R, Esmaeilzadeh A. Solid tumors challenges and new insights of CAR T cell engineering. Stem Cell Rev Rep. (2019) 15:619-36. doi: 10.1007/s12015-019-09901-7

4. Mulazzani M, Frassle SP, von Mucke-Heim I, Langer S, Zhou X, IshikawaAnkerhold $\mathrm{H}$, et al. Long-term in vivo microscopy of CAR T cell dynamics during eradication of CNS lymphoma in mice. Proc Natl Acad Sci USA. (2019) 116:24275-84. doi: 10.1073/pnas.1903854116 inflammatory reactions in the central nervous system (8, 9). Additionally, high-dose intravenous infusion $\left(1 \times 10^{10}\right.$ $\mathrm{T}$ cells) increases the risk of serious pulmonary toxicities (10). Intracerebral delivery may reduce the risk of systemic toxicities and be more suitable for the treatment of PCNSL, a possibility that requires clinical investigation. Additionally, although understanding CAR T-cell dynamics within solid tumors and lymph nodes is important, it is highly desirable to correlate intratumoral and systemic CAR T-cell kinetics in a clinically relevant model, owing to limitations when obtaining serial biopsy specimens from patients.

The findings presented by the authors merit commendation for providing a framework for future investigation into the treatment of PCNSL as well as solid tumors.

\section{AUTHOR CONTRIBUTIONS}

$\mathrm{HH}, \mathrm{HQ}$, and PA wrote the draft. PA reviewed, corrected, and approved the final manuscript. All authors contributed to the article and approved the submitted version.

\section{FUNDING}

PA's laboratory work was supported by grants from the National Institutes of Health (P30 CA008748, R01 CA236615-01, and R01 CA235667), the U.S. Department of Defense (BC132124, LC160212, CA170630, and CA180889), the Batishwa Fellowship, the Comedy vs. Cancer Award, the Druckenmiller Center for Lung Cancer Research Fellowship, the Emerson Collective Cancer Research Fund, the Esophageal Cancer Education Fund, the Memorial Sloan Kettering Technology Development Fund, the Miner Fund for Mesothelioma Research, the Mr. William H. Goodwin and Alice Goodwin, the Commonwealth Foundation for Cancer Research, and the Experimental Therapeutics Center of Memorial Sloan Kettering Cancer Center.

\section{ACKNOWLEDGMENTS}

The authors thank Moira E. McGrath for her editorial assistance.

5. Adusumilli PS, Cherkassky L, Villena-Vargas J, Colovos C, Servais E, Plotkin J, et al. Regional delivery of mesothelin-targeted CAR T cell therapy generates potent and long-lasting CD4-dependent tumor immunity. Sci Transl Med. (2014) 6:261ra151. doi: 10.1126/scitranslmed.3010162

6. Cherkassky L, Morello A, Villena-Vargas J, Feng Y, Dimitrov DS, Jones $\mathrm{DR}$, et al. Human CAR $\mathrm{T}$ cells with cell-intrinsic PD-1 checkpoint blockade resist tumor-mediated inhibition. J Clin Invest. (2016) 126:3130-44. doi: 10.1172/JCI83092

7. Grosser R, Cherkassky L, Chintala N, Adusumilli PS. Combination immunotherapy with CAR $\mathrm{T}$ cells and checkpoint blockade for the treatment of solid tumors. Cancer Cell. (2019) 36:471-82. doi: 10.1016/j.ccell.2019.09.006

8. Akhavan D, Alizadeh D, Wang D, Weist MR, Shepphird JK, Brown CE. CAR T cells for brain tumors: lessons learned and road ahead. Immunol Rev. (2019) 290:60-84. doi: 10.1111/imr.12773

9. Gust J, Hay KA, Hanafi LA, Li D, Myerson D, Gonzalez-Cuyar LF, et al. Endothelial activation and blood-brain barrier disruption in neurotoxicity after adoptive immunotherapy with CD19 CAR-T 
cells. Cancer Discov. (2017) 7:1404-19. doi: 10.1158/2159-8290.CD-1 7-0698

10. Morgan RA, Yang JC, Kitano M, Dudley ME, Laurencot CM, Rosenberg SA. Case report of a serious adverse event following the administration of $\mathrm{T}$ cells transduced with a chimeric antigen receptor recognizing ERBB2. Mol Ther. (2010) 18:843-51. doi: 10.1038/mt.2010.24

Conflict of Interest: PA has received research funding from ATARA Biotherapeutics and OSE Immunotherapies, has received research fees from ATARA Biotherapeutics, and has an issued patent 10,538,588 (mesothelintargeted chimeric antigen receptor) licensed to ATARA Biotherapeutics, issued patent EP1979000B1 (method for detection of cancer cells using virus), and pending patent applications WO2018165228A1, CA3034691A1, CA3007980A1, AU2016316033A1 (PD-1 dominant negative receptor), US20170172477A1 (wireless pulse-oximetry device), and on an ex vivo malignant pleural effusion culture system.

The remaining authors declare that the research was conducted in the absence of any commercial or financial relationships that could be construed as a potential conflict of interest.

Copyright (c) 2020 Hocine, Quach and Adusumilli. This is an open-access article distributed under the terms of the Creative Commons Attribution License (CC BY). The use, distribution or reproduction in other forums is permitted, provided the original author(s) and the copyright owner(s) are credited and that the original publication in this journal is cited, in accordance with accepted academic practice. No use, distribution or reproduction is permitted which does not comply with these terms. 